\title{
Synthesis of Nonporous Poly $(N$-alkylacrylamide) Gel Beads by Nonaqueous Sedimentation Polymerization
}

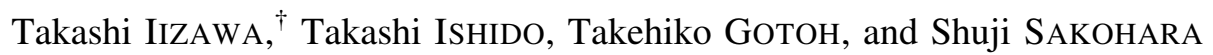 \\ Department of Chemical Engineering, Graduate School of Engineering, Hiroshima University, \\ Kagamiyama, Higashi-Hiroshima 739-8527, Japan
}

(Received August 22, 2006; Accepted October 15, 2006; Published November 24, 2006)

\author{
KEY WORDS Sedimentation Polymerization / Nonporous Beads / Swelling / Thermo-Sensitive \\ Polymer Gel / Poly $(N$-alkylacrylamide) Gel / \\ [doi:10.1295/polymj.PJ2006097]
}

\begin{abstract}
Sedimentation polymerization has attracted much attention as a new method for synthesizing polymer beads because this method is very practical for preparing millimeter-sized monodisperse beads. Polymerization has been carried out using a long cylindrical reactor containing a heating medium such as paraffin oil, ${ }^{1}$ supercritical carbon dioxide, ${ }^{2}$ or silicone oil ${ }^{3}$ and the thermal polymerization of aqueous monomer droplets descending in the medium results in the formation of polymer beads. This method can be extended to the polycondensation of tetraethylorthosilicate in hydrochloric acid ${ }^{4}$ and the crosslinking reaction of poly(vinyl alcohol) with glutalaldehyde ${ }^{5}$ as well as the radical polymerization of vinyl monomers. However, not all monomers or intermediates can be used; they must be water-soluble and must have a high polymerizability. Accordingly, slightly water-soluble or water-insoluble monomers cannot be utilized in this method. In addition, nonporous poly( $N$-isopropylacrylamide) (PNIPA) beads have not been prepared by this method, although the sedimentation polymerization of $N$-isopropylacrylamide (NIPA) using $20 \mathrm{wt} \%$ aqueous $\mathrm{N}, \mathrm{N}$-dimethylformamide (DMF) solution as a solvent resulted in the formation of highly porous PNIPA beads. ${ }^{6,7}$
\end{abstract}

On the other hand, aprotic polar solvents such as sulfolane ${ }^{8}$ and $N$-methyl-2-pyrolidinone (NMP) are well-known extractants used in the separation of aromatic compounds from paraffin oil in the BTX (benzene-toluene-xylene) production process. The abovementioned solvents are good solvents for many organic compounds and polymers, and are immiscible with the heating medium. If these solvents were to be used in sedimentation polymerization instead of aqueous solvents, millimetersized monodisperse polymer beads would be obtained by the polymerization of a variety of monomers such as slightly water-soluble or water-insoluble monomers.

This communication reports a novel nonaqueous sedimentation polymerization using NMP as the solvent; it also reports the successful synthesis of nonporous polymer gel beads from the sedimentation polymerization of $N$-alkylacrylamides such as NIPA, $N, N$-diethylacrylamide (NNDEA), $N$ - $n$-propylacrylamide (NNPA), and $N$ - $t$-butylacrylamide (NTNA).

\section{EXPERIMENTAL}

The typical sedimentation polymerization of NIPA is as fol- lows. NIPA (5.0 g, $44.2 \mathrm{mmol}), N, N^{\prime}$-methylenebisacrylamide (MBAA: $81 \mathrm{mg}, 0.44 \mathrm{mmol}$ ), and $\alpha, \alpha^{\prime}$-azobisisobutyronitrile (AIBN: $50 \mathrm{mg}$ ) were dissolved in NMP $(5.0 \mathrm{~g})$ at $20^{\circ} \mathrm{C}$ in a nitrogen atmosphere. Silicone oil (GE Toshiba Silicones Co., Ltd., TSF451-3000, kinematic viscosity: $3000 \mathrm{~mm}^{2} \mathrm{~s}^{-1}$ at $25^{\circ} \mathrm{C}$ ) was charged in a washing bottle in a water bath at $80^{\circ} \mathrm{C}$, and nitrogen gas was bubbled through the silicone oil for over $3 \mathrm{~h}$. The monomer solution was injected dropwise into the silicone oil via a syringe with a needle (external diameter $1.5 \mathrm{~mm}, 90^{\circ} \mathrm{tip}$ ). The droplets at the base were allowed to stand for $12 \mathrm{~h}$ at $80^{\circ} \mathrm{C}$ under a stream of nitrogen. The resulting beads were washed with methanol in a Soxhlet extractor and dried in vacuo at $60^{\circ} \mathrm{C}$ to constant weights. The average diameter $\left(\overline{2 R_{0}}\right)$ and average weight $(\bar{w})$ of a typical bead were $2.06 \mathrm{~mm}$ (relative standard deviation: $6.42 \%$ ) and $5.02 \mathrm{mg}$, respectively.

The swelling rate of the beads was measured as follows. A bead was placed in water at $20^{\circ} \mathrm{C}$. The change in the external radius $\left(R_{\mathrm{t}} / R_{0}\right)$, where $R_{0}$ and $R_{\mathrm{t}}$ are the external radii of the dried bead and the bead swollen in water for t min, respectively, was periodically recorded by means of a digital video camera (Sony DCR-HC1000).

\section{RESULTS AND DISCUSSION}

The nonaqueous sedimentation polymerization of a NIPA solution containing $1 \mathrm{wt} \%$ AIBN as the radical initiator was carried out using a simple sedimentation polymerization apparatus consisting of a $500 \mathrm{~mL}$ gas-washing bottle of height $15 \mathrm{~cm}$ and highly viscous silicone oil (kinematic viscosity: $3000 \mathrm{~mm}^{2} \mathrm{~s}^{-1}$ at $25^{\circ} \mathrm{C}$ ) as the heating medium in a water bath at $80^{\circ} \mathrm{C}$, as reported previously..$^{3,6,7}$ The results and conditions are summarized in Table I. The sedimentation polymerization mechanism can be described as follows. When millimetersized droplets of the monomer solution descend in the heating medium, heating initially causes gelation of the droplet surface. Thus, stable skins are formed and prevent coalescence of the droplets at the base of the reactor. The polymerization is completed after the droplets reach the base. Accordingly, the polymerization requires sufficient sedimentation time in order to form a stable skin. The sedimentation time can be easily adjusted by the viscosity of the heating medium and the difference between the densities $(d)$ of the monomer solution (sol-

${ }^{\dagger}$ To whom correspondence should be addressed (Tel: +81-82-424-7711, Fax: +81-82-424-5494, E-mail: tiizawa@ hiroshima-u.au.jp). 
Table I. Synthesis of gel beads by sedimentation polymerization ${ }^{\mathrm{a}}$

\begin{tabular}{lllccc}
\hline Gel & \multicolumn{1}{c}{$\begin{array}{c}\text { Monomer } \\
(\mathrm{wt} \%)\end{array}$} & Solvent & $\begin{array}{c}\text { Conc. of MBAA } \\
(\mathrm{mol} \%)\end{array}$ & $\begin{array}{c}\overline{2 R_{0}} \mathrm{~b} \\
(\mathrm{~mm})\end{array}$ & $\begin{array}{c}\bar{w}^{\mathrm{c}} \\
(\mathrm{mg})\end{array}$ \\
\hline G-1 & NIPA (55) & NMP & 2.0 & 2.15 & 6.08 \\
G-2 & NIPA (55) & NMP & 1.0 & 2.08 & 5.33 \\
G-3 & NIPA (55) & NMP & 0.5 & $-\mathrm{e}$ & \\
G-4 & NIPA (55) & NMP & 0.3 & $-\mathrm{f}$ & \\
G-5 & NIPA (50) & NMP & 1.0 & 2.06 & 5.12 \\
G-6 & NIPA (50) & NMP & 0.5 & $-\mathrm{f}$ & \\
G-7 & NIPA (40) & NMP & 1.0 & - f $^{-}$ & \\
G-8 & NIPA (30) & NMP & 1.0 & $-{ }^{\mathrm{f}}$ & \\
G-9 & NIPA (20) & water & 1.0 & $2.50^{\mathrm{d}}$ & 2.66 \\
G-10 & NNPA (55) & NMP & 1.0 & 2.22 & 6.35 \\
G-11 & NNDEA (55) & NMP & 1.0 & 2.25 & 6.47 \\
G-12 & NTBA-NNPA (55) & NMP & 1.0 & 2.26 & 6.81 \\
\hline
\end{tabular}

${ }^{\mathrm{a}}$ Sedimentation polymerization of a NIPA solution $(10 \mathrm{~g})$ was carried out at $80^{\circ} \mathrm{C}$. ${ }^{\mathrm{b}}$ Average diameter of a dried bead. ${ }^{\mathrm{c}}$ Average weight of a dried bead. ${ }^{\mathrm{d}}$ Average diameter of a bead shrunk in water at $80^{\circ} \mathrm{C}$. ${ }^{\mathrm{e}}$ Distorted beads were obtained. ${ }^{\mathrm{f}}$ Beads were not obtained. ${ }^{\mathrm{g}}$ Mixture of $50 \mathrm{~mol} \% \mathrm{NTBA}$ and $50 \mathrm{~mol} \% \mathrm{NNPA}$.

vent) and the heating medium because the settling velocity of the particles obeys Stokes' law. It is important to select a solvent that is immiscible with and slightly heavier than the heating medium, for example, silicone oil $\left(d: 0.971 \mathrm{~g} \mathrm{~mL}^{-1}\right)$. In this study, NMP $\left(d: 1.028 \mathrm{~g} \mathrm{~mL}^{-1}\right)$ was selected, although many aprotic polar solvents with varying densities, such as $N, N$-dimethyacetamide $\left(d: 0.937 \mathrm{~g} \mathrm{~mL}^{-1}\right)$, DMF ( $d: 0.944$ $\left.\mathrm{g} \mathrm{mL}^{-1}\right)$, NMP, dimethylsulfoxide $\left(d: 1.10 \mathrm{~g} \mathrm{~mL}^{-1}\right)$, and sulfolane $\left(d: 1.261 \mathrm{~g} \mathrm{~mL}^{-1}\right)$ have been widely used. A droplet (diameter: approximately $3 \mathrm{~mm}$ ) of the monomer solution sedimented into the silicone oil, and it took about $14 \mathrm{~min}$ to reach the bottom of the apparatus. When the NIPA and crosslinker concentrations in the solution were low to the extent that a stable skin could not be formed, the droplets on the bottom gradually agglutinated and were finally transformed into a polymer mass. When the concentration of NIPA was greater than $50 \mathrm{wt} \%$ and the concentration of the crosslinker was $1 \mathrm{~mol} \%$, polymer beads were obtained. In the polymerization of NIPA, the silicone oil used as a heating medium did not become cloudy due to the dispersion of the minute particles resulting from polymerization. Therefore, polymerization occurred preferentially in the droplets when NMP was used as the solvent, since NIPA is not soluble in silicone oil. The values of $\overline{2 R_{0}}$ and $\bar{w}$ for a dried PNIPA bead are approximately 2.0-2.2 $\mathrm{mm}$ and 5-6 $\mathrm{mg}$, respectively, and are directly dependent on the monomer concentration.

The typical photographs of G-5 beads are shown in comparison with the porous beads (G-9) obtained from the conventional polymerization of a NIPA solution in $20 \mathrm{wt} \%$ aqueous
DMF solution (Figure 1) ${ }^{6,7}$ The G-9 beads that shrunk in water at $80^{\circ} \mathrm{C}$ were used because they were highly distorted after drying due to their complex structure. 6,7 The G-5 beads are transparent, while the shrunken G-9 beads are opaque, and both these beads are spherical in shape. The value of $\bar{w}$ for a dried G-5 bead is much larger than that for a shrunken G-9 bead; however, the average diameter of the former is considerably smaller than that of the latter. The density, as calculated from the values of $\bar{w}$ and $\overline{2 R_{0}}$ of $\mathrm{G}-5$, is approximately 1.1 $\mathrm{g} \mathrm{mL}^{-1}$. This value is in agreement with that previously reported for soluble PNIPA, namely, $1.070 \mathrm{~g} \mathrm{~mL}^{-1} .{ }^{9}$ The relative standard deviation of the diameter of the dried G-5 beads and shrunken G-9 beads is 6.42 and $3.26 \%$, respectively. Although the value of the G-5 beads is slightly higher than that of the G-9 beads, these values are almost the same for the beads that are prepared using a syringe, as reported previous$1 y^{3,6}$ and by Ruckenstein et al. ${ }^{1}$ Therefore, the beads prepared here have a narrow particle size distribution after drying. These results indicate that the polymerization of NIPA in NMP proceeded homogeneously to yield transparent nonporous PNIPA gel beads. Here, the phase separation polymerization occurred at a temperature higher than the lower critical solution temperature (LCST) for water or aqueous solvent to yield opaque porous gels. ${ }^{6,7,10-12}$

The polymerization of a few $\mathrm{N}$-alkylacrylamides was conducted with the monomer concentration and the MBAA content fixed at $55 \mathrm{wt} \%$ and $1.0 \mathrm{~mol} \%$, respectively. The solubilities of $\mathrm{N}$-alkylacrylamides and the corresponding polymers change dramatically with their alkyl groups. For example, NNDEA has properties that are similar to those of NIPA, which is also water-soluble; however, the resulting polymer is insoluble in hot water at temperatures above $32{ }^{\circ} \mathrm{C} .{ }^{13} \mathrm{NNPA}$ is slightly soluble in water, and the resulting polymer is insoluble in water at temperatures above $21^{\circ} \mathrm{C}$. ${ }^{13,14}$ NTBA and the resulting polymer are insoluble in water. NTBA is not very soluble in NMP and beads cannot be only prepared by the polymerization of NTBA alone; therefore, a monomer mixture of NTBA and NNPA (1:1) was used. However, the $N$-alkylacrylamides and NNDEA used are barely soluble in silicone oil, and polymerization proceeded smoothly, resulting in the formation of transparent polymer beads, which cannot be obtained by the conventional sedimentation polymerization using an aqueous solvent. On the other hand, in the case of monomers such as $t$-butyl acrylate and 2-phenoxyethy acrylate that are freely soluble in silicone oil as well as NMP, polymerization occurred mainly in the silicone oil and no beads were obtained. These results indicate that this method is useful for the synthesis of millimeter-sized polymer beads from the polymerization of $\mathrm{N}$-alkylacrylamides with a large partition coefficient between the NMP and silicon oil.
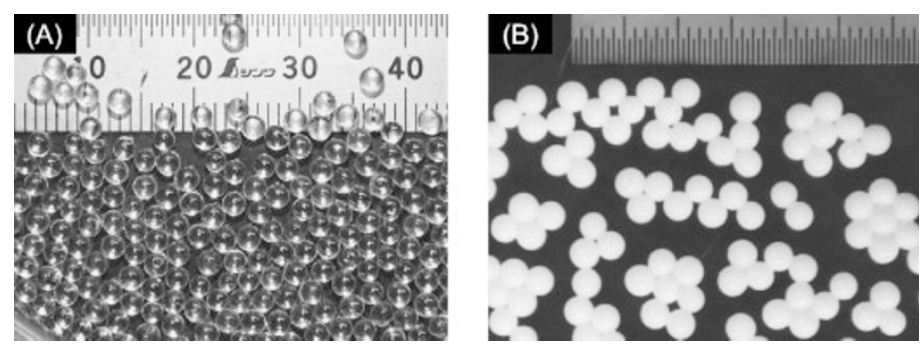

Figure 1. Typical photographs of PNIPA beads produced in this study: (A) dried G-5 beads and (B) shrunken G-9 beads. 


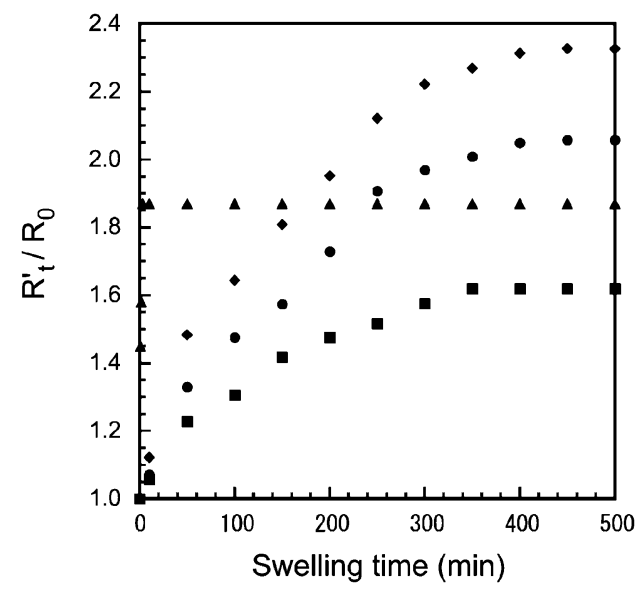

Figure 2. Swelling of PNIPA beads in water at $20^{\circ} \mathrm{C}: \square \mathrm{G}-1, \bigcirc \mathrm{G}-2$, - G-5, $\Delta \mathrm{G}-9$.

The PNIPA gel has a LCST at $32-4{ }^{\circ} \mathrm{C} .{ }^{15-17}$ The PNIPA beads produced by this method did not swell in water at temperatures above the LCST. When the PNIPA gels $\left(2 R_{0}=\right.$ ca. $2.0-2.2 \mathrm{~mm}$ ) were dipped in water at $20^{\circ} \mathrm{C}$, it resulted in swelling, as shown in Figure 2. The swelling was evaluated by the $R_{\mathrm{t}} / R_{0}^{\prime}$ ratio, where $R_{0}^{\prime}$ and $R_{\mathrm{t}}$ are the external radii of the shrunken G-9 beads and the gels swollen in water for t min, respectively. The swelling ratio increased with the decrease in the monomer and crosslinker concentrations. A remarkable feature of the PNIPA beads produced by this method is that the swelling occurs much slower than in the case of the porous G-9 beads. The PNIPA beads swelled very slowly but maintained their spherical shape and attained equilibrium after 450 min, whereas a G-9 bead underwent rapid swelling attaining equilibrium within $4 \mathrm{~min}$. The applications of the PNIPA gel depend on its shape, particle size, and internal structure. The resulting nonporous millimeter-sized PNIPA gels are expected to be useful as drug carriers for long-term chemical release or as returnable water-absorbing polymers for the separation of relatively large molecules and polymers from dilute aqueous solutions. ${ }^{18}$

From these results, we conclude that the nonporous millimeter-sized poly $(N$-alkylacrylamide $)$ beads can be easily ob- tained by nonaqueous sedimentation polymerization when NMP is used as the solvent. We believe that this method of synthesizing millimeter-sized beads may have wide applications for a variety of monomers with a large partition coefficient between NMP and the silicon oil. Furthermore, the scope of applications could be extended by further improvements in the nonaqueous sedimentation polymerization conditions used.

Further studies on the nonaqueous sedimentation polymerization of various monomers and the applications of the resulting beads are currently in progress.

Acknowledgment. The authors thank the Regional Research \& Development Consortium Project by the Chugoku Bureau of Economy, Trade and Industry for their financial support.

\section{REFERENCES}

1. E. Ruckenstein and I. Hong, Polymer, 36, 2857 (1995).

2. H. Zheng and A. I. Cooper, Macromolecules, 36, 5061 (2003).

3. T. Iizawa, K. Nakao, T. Yamaguchi, and M. Maruta, Polymer, 46, 1834 (2005).

4. E. Ruckenstein and I. Hong, J. Appl. Polym. Sci., 61, 1949 (1996).

5. E. Ruckenstein and I. Hong, Chem. Mater., 8, 546 (1996).

6. T. Iizawa, T. Ninomiya, T. Gotoh, and S. Sakohara, Polym. J., 36, 356 (2004).

7. T. Iizawa, H. Taketa, M. Maruta, T. Ishido, T. Gotoh, and S. Sakohara, J. Appl. Polym. Sci., in press.

8. M. N. Papdopolos and C. H. Peal Jr., U. S. Patent 2,999,892 (1958).

9. H. W. Coover Jr., J. Polym. Sci., 39, 532 (1959).

10. B. G. Kabra and S. H. Gehrke, Polym. Commun., 32, 322 (1991).

11. X. S. Wu, A. S. Hoffman, and P. Yager, J. Polym. Sci., Part A: Polym. Chem., 30, 2121 (1992).

12. T. Gotoh, Y. Nakatani, and S. Sakohara, J. Appl. Polym. Sci., 69, 895 (1998).

13. S. Ito, Kobunshi Ronbunshu, 46, 437 (1989).

14. T. Iizawa, Y. Matsuura, and Y. Onohara, Polymer, 46, 8098 (2005).

15. M. Heskins and J. E. Guillet, J. Macromol. Sci. Chem., A2, 1441 (1968).

16. S. Hirotsu, Y. Hirokawa, and T. Tanaka, J. Chem. Phys., 87, 1392 (1987).

17. S. Fujishige, K. Kubota, and I. Ando, J. Phys. Chem., 93, 3311 (1989).

18. N. Kayaman, D. Kazan, A. Erarslan, O. Okay, and B. M. Baysal, J. Appl. Polym. Sci., 67, 805 (1998). 